\title{
MPP01, a new solution for planetary perturbations in the orbital motion of the Moon
}

\author{
P. Bidart \\ DANOF - UMR 8630, Observatoire de Paris, 61 avenue de l'Observatoire, 75014 Paris, France \\ e-mail: bidart@danof .obspm.fr \\ Received 18 May 2000 / Accepted 3 November 2000

\begin{abstract}
We present in this paper MPP01, a new solution for the planetary perturbations in the orbital motion of the Moon. Comparisons of the semi-analytical solution ELP2000-82B to numerical integrations of the Jet Propulsion Laboratory and to LLR observations have shown deficiencies resulting mainly from planetary perturbations series established twenty years ago. The publication of a new planetary solution VSOP2000 and the progresses in numerical tools have contributed to improve the precision of the MPP01 solution. Comparisons between MPP01 and the ELP2000-82B solution for planetary perturbations show differences which are mainly due to the long periodic terms of the solution.
\end{abstract}

Key words. celestial mechanics, stellar dynamics - Moon - solar system: general

\section{Introduction}

Since 1996, the Lunar Laser Ranging at CERGA (Centre d'Études et de Recherche en Géodynamique et Astronomie) provides lunar observations with a precision under the centimeter level in the distance. Using a complete model including the ELP2000-82B solution for the lunar orbital motion, the residuals are estimated roughly to $3 \mathrm{~cm}$ in $\mathrm{rms}$.

Nevertheless the main limitation in the precision in ELP solution results from planetary perturbations whose precision is around few meters in rms. Hence, numerical complements are introduced to improve the solution and to allow comparisons with observations. The aim of this work is to improve the accuracy of the planetary perturbations component in order to reduce the contribution of these complements and to enlarge the timescale validity of the solution providing series expanded up to the fifth power of the time. It has been possible thanks to the publication of a recent planetary solution VSOP2000 by (Moisson 2000). Besides, progresses in numerical tools allow us to handle large series with a great precision.

In the first part of this paper, we desbribe the main features of the ELP planetary perturbations theory, and in a second part, we present the algorithms used for the numerical computations and the methods to handle large series necessary to ephemerides computation. Finally, we compare our series to the ELP ones and we exhibit the largest differences arising from long periodic terms, as we expected using a new planetary solution.

\section{Statement of the problem}

\subsection{Description of ELP theory}

The ELP lunar solution comes from the theory elaborated by (Chapront-Touzé \& Chapront 1980). They took their inspiration from Brown's theory. The construction of this theory is divided in two fundamental steps.

In a first step, one considers the system restricted to the three point masses, the Sun (S), the Earth (E) and the Moon $(\mathrm{M})$ called "main problem", taking into account that the Sun's orbit around the Earth-Moon barycenter is Keplerian. The solution of this problem provides us an intermediate solution called main solution (ChaprontTouzé 1980).

In a second step, all the other effects are regarded as pertubations to the main solution: Earth and Moon figures, planetary perturbations, relativity and tides. In this paper, we limit ourselves to the computation of the planetary perturbations in the orbital motion.

\subsection{Main problem solution}

We refer to Chapront-Touzé (1980) in particular for the definition of the constants used. The series provided solving the main problem's equations in spherical ecliptic coordinates $(V, U, R)$ take the general form:

$$
\begin{aligned}
w_{1} \delta_{V}+ & \sum_{i_{1}, \ldots, i_{p}}\left(A_{i_{1}, \ldots, i_{p}}+\sum_{j=1}^{6} B_{i_{1}, \ldots, i_{p}}^{j} \delta z_{j}^{0}\right) \\
& \times \sin \left(i_{1} \lambda_{1}+\cdots+i_{p} \lambda_{p}+\Phi_{i_{1}, \ldots, i_{p}}\right)
\end{aligned}
$$




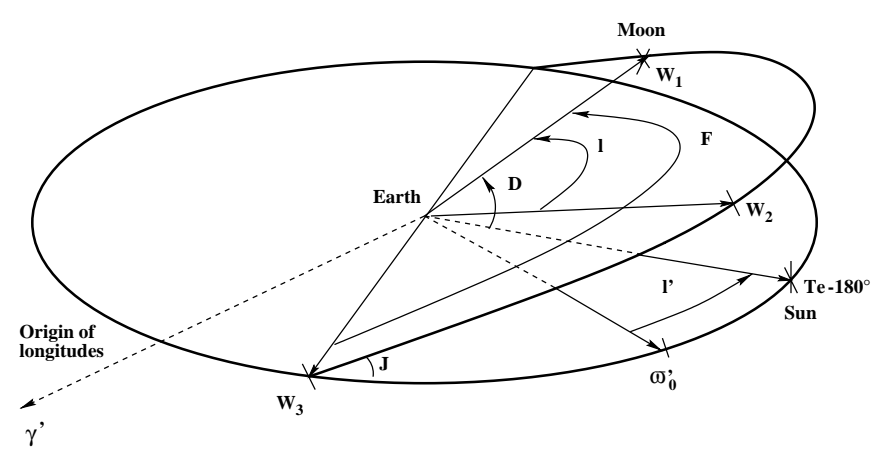

Fig. 1. Delaunay arguments with respect to $w_{j}$

where $\delta_{V}=1$ for the longitude $V$ and $\delta_{V}=0$ otherwise. $A_{i_{1}, \ldots, i_{p}}$ are numerical coefficients and $\Phi_{i_{1}, \ldots, i_{p}}$ are numerical phases. $B_{i_{1}, \ldots, i_{p}}^{j}$ are the first derivatives of $A_{i_{1}, \ldots, i_{p}}$ with respect to the constants used. A first set of constants includes the arbitrary constants of the main problem:

$z_{1}^{0}=\frac{n^{\prime}}{\nu}, z_{2}^{0}=\Gamma, z_{3}^{0}=E$

where $\nu$ and $n^{\prime}$ are respectively the sideral mean motion of the Moon and of the Sun, $\Gamma$ the half coefficient of the $\sin l$ term in longitude, and $E$ the half coefficient of the $\sin F$ term in latitude. The others are $e^{\prime}$, the solar eccentricity, $\alpha$, the ratio of the semi-major axis of the Moon over the Sun's one, and the masses of the Moon $m_{\mathrm{M}}$ and the Earth $m_{\mathrm{E}}$ through the ratio $\mu=m_{\mathrm{M}} /\left(m_{\mathrm{E}}+m_{\mathrm{M}}\right) . \delta z_{j}^{0}$ are the literal linear variations of the three arbitrary constants.

The component $\lambda_{j}$ in the argument stands for $T$, the heliocentric mean longitude of the Earth-Moon barycenter, $\varpi_{0}^{\prime}$, the longitude of the perihelion of the Earth-Moon barycenter restricted to the constant part and the three angular variables $w_{j}=w_{j}^{1} t+w_{j}^{0}$ :

$w_{1}$, the mean longitude of the Moon;

$w_{2}$, the secular part of the longitude of the lunar perigee;

$w_{3}$, the secular part of the longitude of the lunar ascending node.

All these arguments are polynomials expanded up to the fourth power of the time. The phases $w_{j}^{0}$ are three other arbitrary constants of the theory. Practically, in the formulation of coordinates, the $\lambda_{j}$ are described with the four Delaunay's arguments $D, F, l, l^{\prime}$. They are derived from $w_{j}, T$ and $\varpi_{0}^{\prime}$ by the relationships (Fig. 1):

$$
\begin{aligned}
D & =w_{1}-T+180^{\circ} \\
F & =w_{1}-w_{3} \\
l & =w_{1}-w_{2} \\
l^{\prime} & =T-\varpi_{0}^{\prime} .
\end{aligned}
$$

\subsection{Planetary solution}

We used in our computations the new planetary solution VSOP2000 established by (Moisson 2000). This solution has been built using the IERS92 set of masses; the constants of integration are fitted on the numerical integration DE403 of the Jet Propulsion Laboratory. It contains perturbations developed up to the eighth order of planetary masses. The accuracy of VSOP2000 with respect to VSOP 82 has been improved roughly by a factor of ten to twenty for inner planets and one hundred for the outer ones. Here we have neglected the effects of asteroids and Pluto on the planets, and truncated the VSOP2000 series to the fifth power of the time. The planetary series take then the form:

$$
\sum_{n=0}^{5} t^{n} \sum_{i_{1}, \ldots, i_{p}} C_{i_{1}, \ldots, i_{p}}^{(n)} \sin \left(i_{1} \lambda_{1}+\cdots+i_{p} \lambda_{p}+\Phi_{i_{1}, \ldots, i_{p}}^{(n)}\right)
$$

where, as in the main problem series, $C_{i_{1}, \ldots, i_{p}}^{(n)}$ are numerical coefficients and $\Phi_{i_{1}, \ldots, i_{p}}^{(n)}$ are numerical phases. The $\lambda_{j}$ are the linear combination of the mean longitudes of the eight planets: $\bar{\lambda}_{j}=N_{j}^{0} t+\lambda_{j}^{0}$. Table 1 shows the integration constants of the planetary solution that we have used in the computation of planetary perturbations of the Moon.

\section{Equations of motion}

\subsection{Reference frame}

We consider the reference frame $\mathcal{R}_{0}$ defined with the inertial mean ecliptic J2000 and the inertial mean equinox $\gamma_{2000}^{\mathrm{I}}$. It is a fixed frame. The ELP moving reference frame $\mathcal{R}$ is defined as follows:

- the reference plane is the inertial mean ecliptic of date;

- the origin of longitudes points towards the point $\gamma_{2000}^{\prime}$ defined such that:

$N \gamma_{2000}^{\prime}=N \gamma_{2000}^{\mathrm{I}}$

where $N$ is the ascending node of the inertial mean ecliptic of date on the inertial mean ecliptic J2000 (Fig. 2). $\gamma_{2000}^{\prime}$ is the non-rotating origin that Brown used in his theory named departure point. The coordinates of the Moon, expressed in the fixed reference frame are deduced from coordinates expressed in the moving reference frame by adding the accumulated precession in longitude $p_{\mathrm{A}}$ between J2000 and the date. The numerical value of the precession $p_{\mathrm{A}}$ that we used in our computations is given in Simon et al. (1994). Each coordinate will hereafter be expressed in the frame $\mathcal{R}$.

\subsection{Equations in $\mathcal{R}$}

Let us consider the four body problem and their masses: Earth $\left(\mathrm{E}, m_{\mathrm{E}}\right)$, Moon $\left(\mathrm{M}, m_{\mathrm{M}}\right)$, Sun $\left(\mathrm{S}, m^{\prime}\right)$ and a planet $\left(\mathrm{P}, m_{\mathrm{P}}\right) . G$ is the Earth-Moon barycenter. We refer to the Fig. 3 for the definition of distances $D$ and $\Delta . \mu$ is the masses ratio: $\mu=\frac{m_{\mathrm{M}}}{m_{\mathrm{E}}+m_{\mathrm{M}}}$. The equations of motion in the Hamiltonian formulation can be written in the system 
Table 1. Integration constants of the VSOP2000 solution fitted to DE403 in (Moisson 1999). Units are radians per thousand of julian years for $N^{0}$ and radians for $\lambda^{0}$

\begin{tabular}{lrrrrrr}
\hline Planet & $N^{0}$ & $\lambda^{0}$ & $k^{0}$ & $h^{0}$ & $q^{0}$ & $p^{0}$ \\
\hline Mercury & 26087.9031405997 & 4.40260863422 & .04466063577 & .20072330414 & .04061565385 & .04563549294 \\
Venus & 10213.2855473855 & 3.17613445715 & -.00449281998 & .00506684832 & .00682411318 & .02882282404 \\
EMB & 6283.0758504457 & 1.75346994632 & -.00374081857 & .01628448821 & .00000000000 & .00000000000 \\
Mars & 3340.6124347175 & 6.20349959869 & .08536559272 & -.03789970867 & .01047042990 & .01228448748 \\
Jupiter & 529.6909721118 & .59954667809 & .04698584885 & .01200370563 & -.00206544402 & .01118380996 \\
Saturn & 213.2990797783 & .87401678345 & -.00296006971 & .05542959194 & -.00871728519 & .01989130917 \\
Uranus & 74.7816656905 & 5.48122762581 & -.04595338320 & .00564804585 & .00185927537 & .00648605543 \\
Neptune & 38.1329181312 & 5.31189410499 & .00599810784 & .00669103056 & -.01029144683 & .01151685345 \\
\hline
\end{tabular}

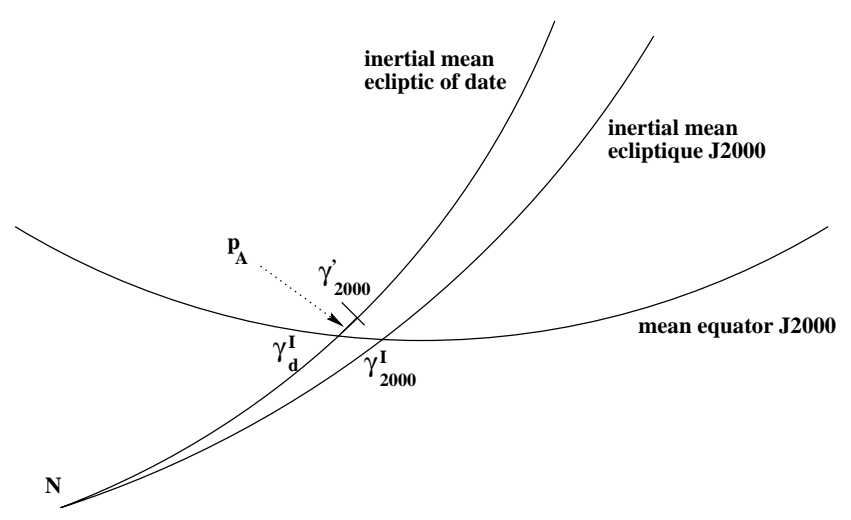

Fig. 2. ELP reference frame

of rectangular coordinates $\left(x_{i} ; u_{i}=\frac{\mathrm{d} x_{i}}{\mathrm{~d} t}\right)$ :

$\mathcal{H}=\frac{1}{2} \sum_{i} u_{i}^{2}-\mathcal{F}-\mathcal{G}$

$\mathcal{G}$ is the force function induced by the rotation of the reference frame, and $\mathcal{F}$ is:

$$
\begin{aligned}
\mathcal{F}=\frac{k\left(m_{\mathrm{E}}+m_{\mathrm{M}}\right)}{r} & +k m^{\prime}\left(\frac{1}{\mu} \frac{1}{\Delta_{2}}+\frac{1}{1-\mu} \frac{1}{\Delta_{1}}\right) \\
& +k m_{\mathrm{P}}\left(\frac{1}{\mu} \frac{1}{D_{2}}+\frac{1}{1-\mu} \frac{1}{D_{1}}\right) .
\end{aligned}
$$

It includes the main problem force function. We divide $\mathcal{F}$ into several components to distinguish the effects that we will take into account:

$\mathcal{F}_{\mathrm{c}}=\frac{k\left(m_{\mathrm{E}}+m_{\mathrm{M}}\right)}{r}+k m^{\prime}\left[\frac{1}{\mu} \frac{1}{\Delta_{2}}+\frac{1}{1-\mu} \frac{1}{\Delta_{1}}\right]_{\mathrm{c}}$

is the force function of the main problem. Index c meaning that the Sun's motion around the center of mass $G$ is Keplerian.

$\mathcal{F}_{\mathrm{I}}=\frac{k\left(m_{\mathrm{E}}+m_{\mathrm{M}}\right)}{r}+k m^{\prime}\left(\frac{1}{\mu} \frac{1}{\Delta_{2}}+\frac{1}{1-\mu} \frac{1}{\Delta_{1}}\right)-\mathcal{F}_{\mathrm{c}}$

is the force function giving rise to indirect perturbations. These perturbations are induced by the whole set of planets on the Moon through the Earth-Moon barycenter.

$\mathcal{F}_{\mathrm{D}}=k m_{\mathrm{P}}\left(\frac{1}{\mu} \frac{1}{D_{2}}+\frac{1}{1-\mu} \frac{1}{D_{1}}\right)$

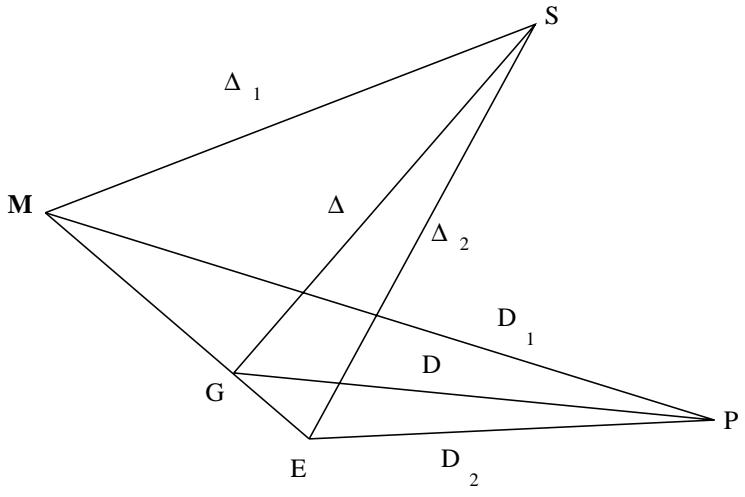

Fig. 3. Schema of the four bodies system

is the force function giving rise to direct perturbations, which are the perturbations induced by a planet acting directly on the Moon. Then, the expression of the Hamiltonian $\mathcal{H}$ becomes:

$\mathcal{H}=H_{\mathrm{c}}-\mathcal{F}_{\mathrm{I}}-\mathcal{F}_{\mathrm{D}}-\mathcal{G}$

with $H_{\mathrm{c}}=\frac{1}{2} \sum_{i} u_{i}^{2}-\mathcal{F}_{\mathrm{c}}\left(x_{i}, t\right)$ describing the main problem. The integration method used to solve this Hamiltonian system is the variation of arbitrary constants.

\section{Integration method}

\subsection{Integration of the Hamiltonian}

The method that we used has been described in (Chapront-Touzé \& Chapront 1980). We briefly summarize the main features of this method. In rectangular coordinates, the equations of motion from the main problem are written:

$\frac{\mathrm{d} x_{i}}{\mathrm{~d} t}=\frac{\partial H_{\mathrm{c}}}{\partial u_{i}}, \quad \frac{\mathrm{d} u_{i}}{\mathrm{~d} t}=-\frac{\partial H_{\mathrm{c}}}{\partial x_{i}}$.

In the main problem, $u_{i}=\frac{\mathrm{d} x_{i}}{\mathrm{~d} t}$. Let $\left(x_{i}^{*}, u_{i}^{*}\right)$ be the solution of the system (8) expressed in Fourier series like (1) whose coefficients are functions of $z_{j}^{0}$ and arguments are linear combinations of $w_{j}$, as we saw in (2.2). Then, we 
have:

$x_{i}=x_{i}^{*}\left(z_{j}^{0} ; w_{j} ; t\right)$

$u_{i}=u_{i}^{*}\left(z_{j}^{0} ; w_{j} ; t\right)$.

By the means of three functions $z_{j}^{0}=z_{j}^{0}\left(c_{k}\right)$ we define a canonical change of coordinates:

$\left(x_{i}, u_{i}\right) \longrightarrow\left(w_{k}, c_{k}\right)$.

Then we consider a new Hamiltonian $\mathcal{H}$ deduced from $H_{\mathrm{c}}$ by:

$\mathcal{H}=H_{\mathrm{c}}-\mathcal{F}_{\mathrm{I}}-\mathcal{F}_{\mathrm{D}}-\mathcal{G}=H_{\mathrm{c}}-\mathcal{P}=\frac{1}{2} \sum_{i} u_{i}^{2}-\left(\mathcal{F}_{\mathrm{c}}+\mathcal{P}\right)$

$\mathcal{P}$ is the disturbing function to be added to the main problem. The system (8) becomes:

$\frac{\mathrm{d} w_{j}}{\mathrm{~d} t}=w_{j}^{(1)}+\left[C^{-1}\right]^{T} \frac{\partial \mathcal{P}}{\partial z_{i}^{0}}$

$\frac{\mathrm{d} z_{j}^{0}}{\mathrm{~d} t}=C^{-1} \frac{\partial \mathcal{P}}{\partial w_{i}}$

$C$ is a $3 \times 3$ matrix function of $z_{j}^{0}$ which is computed with the main problem's values. The order of magnitude of the function $\mathcal{P}$ is small enough to apply an iterative process for the integration. The numerical values for $z_{j}^{0}$ and $w_{j}$ given by the main solution, that we note $\left(z_{j, \mathrm{c}}^{0}, w_{j, \mathrm{c}}\right)$, are substituted in the function $\mathcal{P}$ before integration. After integration of the three last equations of the system (9) we obtain:

$$
\begin{aligned}
z_{j}^{0} & =z_{j, \mathrm{c}}^{0}+\delta z_{j, \mathrm{c}}^{0}+\left(\int C^{-1}\left(\frac{\partial \mathcal{P}}{\partial w_{i}}\right) \mathrm{d} t\right)_{j} \\
& =z_{j, \mathrm{c}}^{0}+\Delta z_{j, \mathrm{c}}^{0}
\end{aligned}
$$

where $\delta z_{j, \mathrm{c}}^{0}$ are the new integration constants introduced for each disturbing function. Substituting these results into the three first equations of the system (9) and considering the increments of $w_{j}^{(1)}$ with respect to $\Delta z_{k}^{0}$, we get:

$$
\begin{aligned}
\frac{\mathrm{d} w_{j}}{\mathrm{~d} t}=w_{j, \mathrm{c}}^{(1)} & +\sum_{k=1}^{3} \frac{\partial w_{j}^{(1)}}{\partial z_{k}^{0}} \delta z_{k, \mathrm{c}}^{0} \\
& +\sum_{k=1}^{3} \frac{\partial w_{j}^{(1)}}{\partial z_{k}^{0}}\left(\int C^{-1}\left(\frac{\partial \mathcal{P}}{\partial w_{l}}\right) \mathrm{d} t\right)_{k} \\
& -\left(\left[C^{-1}\right]^{T}\left(\frac{\partial \mathcal{P}}{\partial z_{i}^{0}}\right)\right)_{j}
\end{aligned}
$$

thus after integration:

$w_{j}=w_{j}^{0}+\delta w_{j}+\sum_{\alpha=1}^{5} w_{j}^{(\alpha)} t^{\alpha}$

where if $\alpha=1$

$w_{j}^{(1)}=w_{j, \mathrm{c}}^{(1)}+\sum_{i=1}^{3} \frac{\partial w_{j}^{(1)}}{\partial z_{i}^{0}} \delta z_{i, \mathrm{c}}^{0}-\left[\left(\left[C^{-1}\right]^{T}\left(\frac{\partial \mathcal{P}}{\partial z_{i}^{0}}\right)\right)_{j}\right]_{t^{1}}$ or if $\alpha \geq 2$

$$
\begin{aligned}
w_{j}^{(\alpha)}=\left[\sum_{i=1}^{3} \frac{\partial w_{j}^{(1)}}{\partial z_{i}^{0}}\right. & \left.\left(\iint C^{-1}\left(\frac{\partial \mathcal{P}}{\partial w_{l}}\right) \mathrm{d} t\right)_{i}\right]_{t^{\alpha}} \\
- & {\left[\int\left(\left[C^{-1}\right]^{T}\left(\frac{\partial \mathcal{P}}{\partial z_{i}^{0}}\right)\right)_{j}\right]_{t^{\alpha}} . }
\end{aligned}
$$

The brackets $[\cdots]_{t^{\alpha}}$ mean the coefficient of the term in $t^{\alpha}$.

\subsection{Constants adjustment}

The introduction of new arbitrary constants $\delta z_{j}^{0}$ allows us to force the solution to be fitted on the set of constants defined for the main problem $(\nu, \Gamma, E)$. Therefore, we evaluate the new arbitrary constants taking into account that the main problem's constants must be unchanged:

$w_{1}^{(1)}=w_{1, \mathrm{c}}^{(1)}=\nu$

according to (2), we have:

$\frac{\partial w_{1}^{(1)}}{\partial z_{1}^{0}}=-\frac{\nu}{z_{1}^{0}} \quad$ and $\quad \frac{\partial w_{1}^{(1)}}{\partial z_{i}^{0}}=0 \quad$ if $i=2,3$

the relation (13) with $j=1$ provides:

$\delta z_{1, \mathrm{c}}^{0}=-\frac{z_{1}^{0}}{\nu}\left[\left(\left[C^{-1}\right]^{T}\left(\frac{\partial \mathcal{P}}{\partial z_{1}^{0}}\right)\right)_{1}\right]_{t^{0}}$.

To obtain the remainder $\delta z_{i}^{0}$, we express the longitude of the Moon:

$$
\begin{aligned}
V & =V_{\mathrm{c}}+\Delta V \\
& =V_{\mathrm{c}}+\sum_{j} \frac{\partial V}{\partial z_{j}^{0}}\left(z_{j}^{0}-z_{j, \mathrm{c}}^{0}\right)+\sum_{j} \frac{\partial V}{\partial w_{j}}\left(w_{j}-w_{j, \mathrm{c}}\right) .
\end{aligned}
$$

The coefficients of the $\sin l$-terms in $V$ and $V_{\mathrm{c}}$ must be equal, hence we have:

$$
\begin{array}{r}
\sum_{j}\left[\frac{\partial V}{\partial z_{j}^{0}}\right]_{\sin l} \delta z_{j}^{0}+\left[\sum_{j} \frac{\partial V}{\partial z_{j}^{0}}\left(\int C^{-1}\left(\frac{\partial \mathcal{P}}{\partial w_{i}}\right) \mathrm{d} t\right)_{j}\right]_{\sin l} \\
+\left[\sum_{j} \frac{\partial V}{\partial w_{j}} \delta w_{j}\right]_{\sin l} .
\end{array}
$$

The adjustment of the $\sin F$-term in the latitude provides another linear equation. Solving this linear system and taking into account the relation (16) one determines the $\delta z_{j}^{0}$.

\section{Algorithm for the left member computation}

\subsection{Direct perturbations}

As shown in Fig. 3, $D_{1}$ and $D_{2}$ occurring in the expression of $\mathcal{F}_{\mathrm{D}}$ (6) can be developed (Chapront \& Abu el Ata 1977) in Legendre polynomials of the variable $\theta_{\mathrm{P}}=\frac{1}{r D} \boldsymbol{T} \boldsymbol{L} \cdot \boldsymbol{G P}$ :

$\mathcal{F}_{\mathrm{D}}=\frac{k m_{\mathrm{p}}}{D} \sum_{n \geq 2} c_{n}\left(\frac{r}{D}\right)^{n} P_{n}\left(\theta_{\mathrm{P}}\right)$ 
where scalars $c_{n}$ are functions of masses:

$c_{n}=(1-\mu)^{n-1}+(-1)^{n} \mu^{n-1}$.

The Legendre polynomials are expanded with an analogous approach to Brown's. One makes a separation of monomials depending on lunar coordinates solely from polynomials referring to planetary elements.

Let $\left(x_{1}, x_{2}, x_{3}\right)$ and $\left(y_{1}, y_{2}, y_{3}\right)$ be respectively the coordinates of the vector $\boldsymbol{T} \boldsymbol{L}$ and of the vector $\boldsymbol{G P}$. We have for $\theta_{\mathrm{P}}$ :

$\theta_{\mathrm{P}}=\frac{x_{1} y_{1}+x_{2} y_{2}+x_{3} y_{3}}{\sqrt{x_{1}^{2}+x_{2}^{2}+x_{3}^{2}} \cdot \sqrt{y_{1}^{2}+y_{2}^{2}+y_{3}^{2}}}$

$\theta_{\mathrm{P}}$ is substituted in (18); we obtain for $\mathcal{F}_{\mathrm{D}}$ :

$\mathcal{F}_{\mathrm{D}}=k m_{\mathrm{P}} \sum_{i \geq 2} \mathcal{L}^{(i)}$

The index $i$ indicates that the expression is homogeneous by a degree $i$ with respect to $x_{j}$. Thus we have:

$\mathcal{L}^{(i)}=\sum_{n_{1}+n_{2}+n_{3}=i} L^{(i)} x_{1}^{n_{1}} x_{2}^{n_{2}} x_{3}^{n_{3}}$

where $L^{(i)}$ are polynomials $\left(X_{j}\right)$ of $y_{i}$ and odd powers of the inverse of the distance $D$ :

$L^{(i)}=\sum_{n \leq i} X_{j}\left(\frac{1}{D^{2 n+1}} \cdot y_{j}^{2 n-2}\right)$.

This separation of coordinates suggested by Brown is extremely important for the integration of the Hamiltonian system. As a matter of fact, the multiplication of series whose arguments are different increases considerably the number of terms. On the other hand, during the integration, most of the short periodic terms vanish whereas small divisors increase the amplitude of long periodic terms. A drastic truncation level in the multiplication would prevent us from obtaining all the significant long periodic terms. The separation of coordinates allows us to accomplish simultaneously the multiplication and the integration avoiding truncation in multiplications alone.

In the equations of system (9) the elements of the $C^{-1}$ matrix are proportional to $1 / \nu a_{0}^{2}$. Here $a_{0}$ is the lunar semi-major axis tied to $\nu$ by: $\nu^{2} a_{0}^{3}=k\left(m_{\mathrm{E}}+\right.$ $m_{\mathrm{M}}$ ). Replacing in (9) $\mathcal{P}$ by $\mathcal{F}_{\mathrm{D}}$ from (18), we get the coefficient:

$\frac{k m_{\mathrm{P}}}{\nu a_{0}^{2}}=\frac{m_{\mathrm{P}}}{m^{\prime}} \frac{n^{\prime 2} a^{\prime 3}}{\nu a_{0}^{2}} \beta$

with $\beta=\frac{m^{\prime}}{m^{\prime}+m_{\mathrm{E}}+m_{\mathrm{M}}}$. Then we have, for instance, the partials of $\mathcal{F}_{\mathrm{D}}$ with respect to the angles $w_{j}$ :

$$
\begin{aligned}
& \nu a_{0}^{2} C^{-1} \frac{\partial}{\partial w_{j}}\left(\frac{\mathcal{F}_{\mathrm{D}}}{\nu a_{0}^{2}}\right)= \\
& \nu a_{0}^{2} C^{-1} \beta \frac{n^{\prime 2}}{\nu} \frac{m_{\mathrm{P}}}{m^{\prime}} \frac{\partial}{\partial w_{j}}\left\{\sum_{i \geq 2}\left(\frac{a_{0}}{a^{\prime}}\right)^{i-2} a^{\prime i+1} \frac{\mathcal{L}^{(i)}}{a_{0}^{i}}\right\}
\end{aligned}
$$

The quantities $a^{\prime i+1} \frac{\mathcal{L}^{(i)}}{a_{0}^{i}}$ are dimensionless and of degree $i$ with respect to the monomials $x_{j} / a_{0}$. Whatever the planet concerned, even for indirect perturbations, lunar coordinates and their partials with respect to $w_{j}$ and $z_{j}^{0}$ are the same. Therefore we construct the above quantities once for all.

\subsection{Indirect perturbations}

Coming back to the disturbing function $\mathcal{F}_{\mathrm{I}}$ in $(5)$, the Sun's orbit is no longer Keplerian but contains the planetary perturbations up to the eighth order of the masses. However, we have to remove the terms which are already computed in the main problem. Hence we compute with a procedure analogous to the direct case, the polynomials in term of the planetary coordinates from VSOP2000 and from their Keplerian expressions. With the VSOP2000 integration constants of the Earth-Moon barycenter (Table 1),

$N^{\prime 0}, \lambda^{\prime 0}, k^{\prime 0}, h^{\prime 0}, q^{\prime 0}, p^{\prime 0}$

we construct the rectangular coordinates from the formulae of Keplerian motion solving Kepler's equation (Chapront et al. 1975). As in the direct case (18), $\mathcal{F}_{\mathrm{I}}$ is developed in Legendre polynomials:

$\mathcal{F}_{\mathrm{I}}=\frac{k m^{\prime}}{r^{\prime}} \sum_{n \geq 2} c_{n}\left(\frac{r}{r^{\prime}}\right)^{n} P_{n}\left(\theta^{\prime}\right)$

where $\theta^{\prime}=\frac{1}{r r^{\prime}} \boldsymbol{T} \boldsymbol{L} \cdot \boldsymbol{G S}$, and $c_{n}$ given in (19). Let $\left(x_{1}^{\prime}, x_{2}^{\prime}, x_{3}^{\prime}\right)$ be the coordinates of the Sun. The separation between the lunar coordinates $\left(x_{j}\right)$ and the solar ones $\left(x_{j}^{\prime}\right)$ leads to an expression similar to $\mathcal{F}_{\mathrm{D}}$ :

$$
\begin{aligned}
\mathcal{F}_{\mathrm{I}} & =k m^{\prime} \sum_{i \geq 2} \mathcal{L}^{\prime(i)} \\
& =k m^{\prime} \sum_{i \geq 2} \sum_{n_{1}+n_{2}+n_{3}=i} L^{\prime(i)} x_{1}^{n_{1}} x_{2}^{n_{2}} x_{3}^{n_{3}}
\end{aligned}
$$

$\mathcal{L}^{\prime(i)}$ are homogenous polynomials of degree $i$ with respect to the coordinates $x_{j}^{\prime}$. We have for $L^{\prime(i)}$ :

$L^{\prime(i)}=L_{\mathrm{VSOP}}^{\prime(i)}-L_{\mathrm{kep}}^{\prime(i)}$

where $L_{\text {VSOP }}^{\prime(i)}\left(\right.$ resp. $\left.L_{\text {kep }}^{\prime(i)}\right)$ have the same expression as in (22) substituting $D$ and $y_{j}$ by $r^{\prime}$ and $x_{j}^{\prime}$ (resp. $r^{\prime(\mathrm{kep})}$ and $\left.x_{j}^{(\mathrm{kep})}\right)$.

\subsection{Perturbations induced by the rotating frame}

The expression of the function $\mathcal{G}$ providing the perturbations induced by the rotating frame has been given in (Chapront-Touzé \& Chapront 1980). We have, in rectangular coordinates:

$\mathcal{G}=\theta_{1}\left(x_{2} u_{3}-x_{3} u_{2}\right)+\theta_{2}\left(x_{3} u_{1}-x_{1} u_{3}\right)$

$+\theta_{3}\left(x_{1} u_{2}-x_{2} u_{1}\right)$ 
$\theta_{i}$ are the components of the instantaneous vector of rotation of $\mathcal{R}$ with respect to $\mathcal{R}_{0}$. One can express the $\theta_{i}$ in terms of the polynomial developments of $p^{\prime}$ and $q^{\prime}$ of the Earth-Moon barycenter and their derivatives with respect to the time:

$$
\begin{aligned}
& \theta_{1}=\frac{2}{\sqrt{1-p^{\prime 2}-q^{\prime 2}}}\left(p^{\prime} q^{\prime} \frac{\mathrm{d} p^{\prime}}{\mathrm{d} t}+\left(1-p^{\prime 2}\right) \frac{\mathrm{d} q^{\prime}}{\mathrm{d} t}\right) \\
& \theta_{2}=\frac{2}{\sqrt{1-p^{\prime 2}-q^{\prime 2}}}\left(\left(1-q^{\prime 2}\right) \frac{\mathrm{d} p^{\prime}}{\mathrm{d} t}+p^{\prime} q^{\prime} \frac{\mathrm{d} q^{\prime}}{\mathrm{d} t}\right) \\
& \theta_{3}=-2\left(q^{\prime} \frac{\mathrm{d} p^{\prime}}{\mathrm{d} t}-p^{\prime} \frac{\mathrm{d} q^{\prime}}{\mathrm{d} t}\right) .
\end{aligned}
$$

With the aid of (28), (27) we integrate the system (9) considering that the coordinates $x_{i}$ and $u_{i}$ are those of the main problem.

\section{Results and comparisons}

\subsection{Timescale and long periodic terms}

Developments as in (23) have been undertaken up to the power $i=4$ for each planet except Venus and Mars. That means we have neglected terms containing the factor $\left(a_{0} a^{\prime}\right)^{3} \sim 1.610^{-8}$. The analogous developments for Venus, Mars, and indirect perturbations have been undertaken up to the power $i=5$ neglecting terms with $\left(a_{0} / a^{\prime}\right)^{4} \sim 410^{-11}$ in factor.

Thanks to the separation of coordinates, we can perform the multiplication of terms weighted by the divisors appearing when integrating the equations. Let $\varepsilon$ be the divisor associated to the argument $\theta$ of a term $A \cos \theta+B \sin \theta$ resulting from a multiplication. During the integration, we keep all the terms with a magnitude $(|A|+|B|) / \varepsilon>\eta$ for $z_{0}^{j}$ and $(|A|+|B|) / \varepsilon^{2}>\eta$ for $w_{j}$, where $\eta$ is the truncation level. We set $\eta \sim 410^{-7^{\prime \prime}}$ in longitude, $\eta \sim 210^{-7^{\prime \prime}}$ in latitude and $\eta \sim 410^{-7}$ kilometers in distance.

Terms whose frequency $\varepsilon$ is of the same order as the Moon's sideral mean motion $\nu$ have a truncation $\eta$. The integration of long periodic terms provides small divisors $\varepsilon \ll \nu$ and even $\varepsilon^{2} \ll \nu$ in the double integration that implies the degradation of the precision by a factor $\frac{\nu}{\varepsilon}\left(\frac{\nu}{\varepsilon^{2}}\right)$. Thus, the precision of computation of long periodic terms depends on the period, and whether it comes from the simple or double integration. In that respect, we neglect terms whose period exceeds 5000 years.

\subsection{Results}

The contribution of planetary perturbations in the final solution occurs through the secular contributions in angular variables $w_{j}(12)$ and through Poisson series formally expressed by $\delta w_{j}(12)$ and $\Delta z_{j}^{0}(10)$. In the series of planetary perturbations, the arguments are linear combinations of the eight planetary mean longitudes and of the four
Delaunay arguments. We can divide those arguments in three categories:

\section{- Lunar short periodic terms}

Combinations of planetary and lunar arguments whose frequency is close to $\nu$. The precision of those terms is the truncation precision $\eta$;

- Planetary long periodic terms combinations of planetary arguments whose frequency is small compared to $\nu$. The precision of those terms coming from the simple integration is the product of precision in the planetary with the factor $\nu / \varepsilon$;

- Lunar long periodic terms combinations of lunar and planetary arguments whose frequency is small compared to $\nu$. The precision of those terms arising from the double integration is the product of main problem's precision with $\nu / \varepsilon^{2}$.

\subsection{Comparisons to ELP2000-82B}

Before any comparisons to the ELP2000-82B solution, one has to take into account the differences in the construction of the previous solution. For indirect perturbations, Taylor developments using increments of the coordinates with respect to the elliptic elements have been used. Here we subtract the Keplerian contribution to the complete expression of the coordinates of the Earth-Moon barycenter. ELP2000-82B has been computed with VSOP82 planetary solution and developed up to the square of the time. In that case, the perturbations include only the secular variations due to solar eccentricity.

Differences in longitude $(\Delta V)$ between the ELP200082B solution for planetary perturbations and MPP01 restricted to Fourier terms produce the results that we expected (Table 3 ). The largest differences are provided by lunar and planetary-type long periodic terms resulting from the computation of $\delta w_{1}(17)$. This effect is mainly explained by the improvement of the planetary solution especially for outer planets whose precision has been improved by two orders of magnitude (Moisson 1999). The numerical tools are more powerful. They allowed us to handle larger series, so that the precision of computation has been increased. The amplitude of such long periodic terms after integration is then better defined.

The terms $(\diamond)$ do not appear in MPP01 whereas the new terms $(*)$ did not appear in ELP2000-82B. The arguments of terms $(\dagger)$ are resulting from combination of long periods. The short periods in the lunar motion should not appear in the difference at this level of precision. But the terms $(\star)$ come from the product of long periods described above with Delaunay arguments in $\partial V / \partial w_{1} \delta w_{1}(17)$. The differences in latitude $(\Delta U)$ and distance $(\Delta R)$ show essentially short periods (Table 4 ). Concerning the Poisson terms for each coordinates, the magnitude of differences does not exceed those of Fourier terms over the time span of two centuries.

In Table 5, we show the main differences in planetary perturbations through the secular contribution in the 
Table 2. Summary of main differences in the construction of ELP2000-82B and MPP01

\begin{tabular}{|c|c|c|}
\hline & ELP2000-82B & MPP01 \\
\hline Indirect perturbations method & $\begin{array}{l}\text { increaments with respect to } \\
\text { elements }\end{array}$ & $\begin{array}{l}\text { complete motion for EMB } \\
\text { minus Keplerian contribu- } \\
\text { tion }\end{array}$ \\
\hline Poisson Series & $\begin{array}{l}\text { development up to } t^{2} \text { (so- } \\
\text { lar eccentricity perturba- } \\
\text { tions only) }\end{array}$ & development up to $t^{5}$ \\
\hline Planetary solution & VSOP82 & VSOP2000 \\
\hline Set of masses & IAU76 & IERS92 \\
\hline Precision of computation & $10^{-5^{\prime \prime}}$ & $4.10^{-7^{\prime \prime}}$ \\
\hline Size of series & 10000 terms & 100000 terms \\
\hline
\end{tabular}

Table 3. Largest differences in longitude between ELP200082B solution for planetary perturbations and MPP01 restricted to Fourier terms. Periods are in julian years, $\Delta A$ are the differences in magnitude expressed in $10^{-3}$ arcsec of degree

\begin{tabular}{lrr}
\hline argument $\omega$ & $\begin{array}{r}\text { period } \\
\text { (year) }\end{array}$ & $\begin{array}{r}\Delta A \\
(\mathrm{mas})\end{array}$ \\
\hline $34 \mathrm{Te}-41 \mathrm{Ma}+2 \mathrm{Ju}-D$ & 1237.472 & 58.65 \\
$15 \mathrm{Ve}-9 \mathrm{Te}-4 \mathrm{Ma}-l$ & 2810.039 & 37.53 \\
$10 \mathrm{Ve}-3 \mathrm{Te}-l$ & 1911.772 & 36.15 \\
$2 \mathrm{Ju}-5 \mathrm{Sa}$ & 883.279 & 25.66 \\
$4 \mathrm{Te}-8 \mathrm{Ma}+3 \mathrm{Ju}$ & 1783.181 & 25.57 \\
$4 \mathrm{Te}-8 \mathrm{Ma}+3 \mathrm{Ju}+l^{\star}$ & 0.075 & 10.35 \\
$4 \mathrm{Te}-8 \mathrm{Ma}+3 \mathrm{Ju}-l^{\star}$ & 0.075 & 10.32 \\
$\mathrm{Te}+\mathrm{Ju}-\mathrm{Sa}+D-F$ & 293.300 & 6.22 \\
$18 \mathrm{Ve}-12 \mathrm{Te}-8 \mathrm{Ma}+3 \mathrm{Ju}-l^{* \dagger}$ & 322.414 & 4.95 \\
$19 \mathrm{Ve}-24 \mathrm{Te}+10 \mathrm{Ju}-D+F-l^{\diamond}$ & 4852.856 & 3.75 \\
$34 \mathrm{Te}-41 \mathrm{Ma}+2 \mathrm{Ju}-D-l^{\star}$ & 0.075 & 3.26 \\
$34 \mathrm{Te}-41 \mathrm{Ma}+2 \mathrm{Ju}-D+l^{\star}$ & 0.075 & 3.26 \\
$20 \mathrm{Ve}-16 \mathrm{Te}+7 \mathrm{Ma}-8 \mathrm{Ju}+6 \mathrm{Sa}-l$ & 272.845 & 3.06 \\
$20 \mathrm{Ve}-12 \mathrm{Te}-15 \mathrm{Ma}-D-F+l$ & 3296.767 & 3.01 \\
$20 \mathrm{Ve}-19 \mathrm{Te}-3 \mathrm{Ju}-l$ & 556.916 & 2.89 \\
$22 \mathrm{Ve}-28 \mathrm{Te}+7 \mathrm{Ma}-2 D+l$ & 640.069 & 2.87 \\
$2 \mathrm{Ju}-5 \mathrm{Sa}-l^{\star}$ & 0.075 & 2.82 \\
$2 \mathrm{Ju}-5 \mathrm{Sa}+l^{\star}$ & 0.075 & 2.82 \\
$4 \mathrm{Te}-8 \mathrm{Ma}+3 \mathrm{Ju}-2 D+l^{\star}$ & 0.087 & 2.80 \\
$4 \mathrm{Te}-8 \mathrm{Ma}+3 \mathrm{Ju}+2 D-l^{\star}$ & 0.087 & 2.78 \\
$18 \mathrm{Ve}-16 \mathrm{Te}-l$ & 273.045 & 2.66 \\
$4 \mathrm{Me}-5 \mathrm{Ve}+3 \mathrm{Te}-2 D+l$ & 1010.584 & 2.54 \\
$\mathrm{Ur}-2 \mathrm{Ne}$ & 4233.468 & 2.28 \\
$10 \mathrm{Ve}-3 \mathrm{Te}{ }^{\star}$ & 0.075 & 2.20 \\
$10 \mathrm{Ve}-3 \mathrm{Te}-2 l^{\star}$ & 0.075 & 2.13 \\
$14 \mathrm{Ve}-9 \mathrm{Te}+2 \mathrm{Ju}-2 F+l$ & 1807.486 & 2.03 \\
$\mathrm{Me}+15 \mathrm{Ve}-16 \mathrm{Te}-D-F+l$ & 1771.543 & 1.99 \\
$15 \mathrm{Ve}-9 \mathrm{Te}-4 \mathrm{Ma}{ }^{\star}$ & 0.075 & 1.99 \\
$15 \mathrm{Ve}-9 \mathrm{Te}-4 \mathrm{Ma}-2 l^{\star}$ & 0.075 & 1.99 \\
\hline & & \\
& & \\
& &
\end{tabular}

angular variables. They are mainly due to the constant adjustment and the indirect perturbations. The modification in VSOP2000 with respect to VSOP87 of planetary constants such as the masses or the mean motions described in (Moisson 1999), explains the small differences observed in the constants adjustment. The difference in the contribution of indirect planetary perturbations is larger than what we expected. It is a consequence of the method used to compute the indirect perturbations. Our method consists in subtracting the contribution of Keplerian
Table 4. Largest differences in latitude and distance between ELP2000-82B solution for planetary perturbations and MPP01 restricted to Fourier terms. $\Delta A$ for distance are the differences in magnitude expressed in meters

\begin{tabular}{|c|c|c|}
\hline \multicolumn{3}{|l|}{ latitude } \\
\hline argument & $\begin{array}{r}\text { period } \\
\text { (year) }\end{array}$ & $\begin{array}{r}\Delta A \\
\text { (mas) }\end{array}$ \\
\hline $34 \mathrm{Te}-41 \mathrm{Ma}+2 \mathrm{Ju}-D-F$ & 0.0745 & 2.61 \\
\hline $34 \mathrm{Te}-41 \mathrm{Ma}+2 \mathrm{Ju}-D+F$ & 0.0745 & 2.61 \\
\hline $15 \mathrm{Ve}-9 \mathrm{Te}-4 \mathrm{Ma}-F-l$ & 0.0745 & 1.66 \\
\hline $15 \mathrm{Ve}-9 \mathrm{Te}-4 \mathrm{Ma}+F-l$ & 0.0745 & 1.66 \\
\hline $10 \mathrm{Ve}-3 \mathrm{Te}-F-l$ & 0.0745 & 1.65 \\
\hline $10 \mathrm{Ve}-3 \mathrm{Te}+F-l$ & 0.0745 & 1.63 \\
\hline $\mathrm{Te}+\mathrm{Ju}-\mathrm{Sa}+D$ & 0.0745 & 1.50 \\
\hline $2 \mathrm{Ju}-5 \mathrm{Sa}+F$ & 0.0745 & 1.02 \\
\hline $2 \mathrm{Ju}-5 \mathrm{Sa}-F$ & 0.0745 & 1.02 \\
\hline \multicolumn{3}{|l|}{ distance } \\
\hline argument & $\begin{array}{r}\text { period } \\
\text { (year) }\end{array}$ & $\begin{array}{r}\Delta A \\
\text { (meter) }\end{array}$ \\
\hline $4 \mathrm{Te}-8 \mathrm{Ma}+3 \mathrm{Ju}+l$ & 0.0754 & 9.49 \\
\hline $4 \mathrm{Te}-8 \mathrm{Ma}+3 \mathrm{Ju}-l$ & 0.0754 & 9.47 \\
\hline $34 \mathrm{Te}-41 \mathrm{Ma}+2 \mathrm{Ju}-D+l$ & 0.0754 & 3.01 \\
\hline $34 \mathrm{Te}-41 \mathrm{Ma}+2 \mathrm{Ju}-D-l$ & 0.0754 & 3.01 \\
\hline $2 \mathrm{Ju}-5 \mathrm{Sa}-l$ & 0.0754 & 2.69 \\
\hline $2 \mathrm{Ju}-5 \mathrm{Sa}+l$ & 0.0754 & 2.69 \\
\hline $4 \mathrm{Te}-8 \mathrm{Ma}+3 \mathrm{Ju}-2 D+l$ & 0.0871 & 2.17 \\
\hline $4 \mathrm{Te}-8 \mathrm{Ma}+3 \mathrm{Ju}+2 D-l$ & 0.0871 & 2.13 \\
\hline $10 \mathrm{Ve}-3 \mathrm{Te}$ & 0.0754 & 2.02 \\
\hline $10 \mathrm{Ve}-3 \mathrm{Te}-2 l$ & 0.0754 & 1.97 \\
\hline $15 \mathrm{Ve}-9 \mathrm{Te}-4 \mathrm{Ma}-2 l$ & 0.0754 & 1.84 \\
\hline $15 \mathrm{Ve}-9 \mathrm{Te}-4 \mathrm{Ma}$ & 0.0754 & 1.83 \\
\hline $10 \mathrm{Te}-19 \mathrm{Ma}+3 \mathrm{Sa}+2 D-l$ & 0.0871 & 1.63 \\
\hline $10 \mathrm{Te}-19 \mathrm{Ma}+3 \mathrm{Sa}-2 D+l$ & 0.0871 & 1.63 \\
\hline $8 \mathrm{Te}-16 \mathrm{Ma}+6 \mathrm{Ju}+l$ & 0.0754 & 1.27 \\
\hline $8 \mathrm{Te}-16 \mathrm{Ma}+6 \mathrm{Ju}-l$ & 0.0754 & 1.27 \\
\hline $10 \mathrm{Te}-19 \mathrm{Ma}+3 \mathrm{Sa}+2 D$ & 0.0404 & 1.23 \\
\hline $10 \mathrm{Te}-19 \mathrm{Ma}+3 \mathrm{Sa}-2 \mathrm{D}$ & 0.0404 & 1.22 \\
\hline $\mathrm{Te}+\mathrm{Ju}-\mathrm{Sa}+D-F+l$ & 0.0755 & 1.03 \\
\hline $\mathrm{Te}+\mathrm{Ju}-\mathrm{Sa}+D-F-l$ & 0.0755 & 1.03 \\
\hline
\end{tabular}

motion in the complete solution of the Earth-Moon barycenter. This method requires a high precision in the computation of Keplerian coordinates. The small contribution of the neglected effects in the fitting of VSOP2000 to the numerical integration DE403 can explain the differences observed. The results concerning the $t^{3}$ (and higher 
Table 5. Differences of the secular contribution in angular variables. Units are arcseconds per century to the power $\alpha$

\begin{tabular}{|c|c|c|c|}
\hline Parameter & & $w_{2}^{(1)}(" 1 / \mathrm{cy})$ & $w_{3}^{(1)}(1 \prime / \mathrm{cy})$ \\
\hline Direct planetary perturbations & & 0.0036 & -0.0013 \\
\hline Indirect planetary perturbations & & -0.0498 & -0.0159 \\
\hline Moon on EMB & & 0.0032 & 0.0002 \\
\hline Relativity & & -0.0002 & 0.0001 \\
\hline Constants adjustment & & -0.0121 & 0.0009 \\
\hline Total & & -0.0553 & -0.0160 \\
\hline Parameter & $w_{1}^{(2)}\left(11 / \mathrm{cy}^{2}\right)$ & $w_{2}^{(2)}\left({ }^{\prime \prime} / \mathrm{cy}^{2}\right)$ & $w_{3}^{(2)}\left({ }^{\prime \prime} / \mathrm{cy}^{2}\right)$ \\
\hline Total & 0.00605 & -0.00135 & -0.00038 \\
\hline
\end{tabular}

Table 6. Comparison of long periodic terms determined by Moshier with those present in MPP01. Periods are in julian years, amplitudes are in arcseconds per century square, and phases are in degrees

\begin{tabular}{lr|rr|rr}
\hline \multicolumn{2}{c|}{ terms in $t^{2}$ sin } & \multicolumn{2}{c|}{ Moshier } & \multicolumn{2}{c}{ MPP01 } \\
$\omega$ & period $(\mathrm{y})$ & $A\left(\left(^{\prime \prime} / \mathrm{cy}^{2}\right)\right.$ & $\Phi\left(^{\circ}\right)$ & $A\left(\left(^{\prime \prime} / \mathrm{cy}^{2}\right)\right.$ & $\Phi\left(^{\circ}\right)$ \\
\hline $18 \mathrm{Ve}-16 \mathrm{Te}-l$ & 273.045 & $-230.310^{-5}$ & 0 & $-228.010^{-5}$ & +23.6 \\
$8 \mathrm{Ve}-13 \mathrm{Te}$ & 238.922 & $-17.710^{-5}$ & +22.5 & $-4.110^{-5}$ & +58.9 \\
$10 \mathrm{Ve}-3 \mathrm{Te}-l$ & 1911.772 & $-30.110^{-5}$ & -60.8 & $-25.410^{-5}$ & -17.3 \\
\hline
\end{tabular}

powers) Poisson terms are not definite. They do not appear in (Table 5).

In the last part of our comparison, we consider the results presented by (Moshier 1992) on a comparison of ELP2000-82B with a lunar ephemeris over a 7000 years time span. To make consistant ELP with the numerical integration on a long time, Moshier adds a quadratic expansion of three long periodic terms and secular contributions in lunar arguments. The effect of these adjustments is to reduce the residuals of the difference in longitude to about $1.5^{\prime \prime} \mathrm{rms}$. The extremum is below $10^{\prime \prime}$ over the 7000 years time span. The comparisons of the long periodic terms of MPP01 with the results obtained by Moshier are shown in Tables 5 and 6 . We observe a good agreement concerning the amplitude of the term $18 \mathrm{Ve}-16 \mathrm{Te}-l$. The differences in the amplitudes of the terms $10 \mathrm{Ve}-3 \mathrm{Te}-l$ and $8 \mathrm{Ve}-13 \mathrm{Te}$ are reduced when we consider the development of these terms in higher powers of time and also the other long periodic terms $(2 \mathrm{Ju}-5 \mathrm{Sa}$ and $4 \mathrm{Te}-8 \mathrm{Ma}+3 \mathrm{Ju})$ which were not mentioned in (Moshier 1992). The comparisons are also in good agreement with a shift of $25^{\circ}$ in phase observed by Moshier concerning terms involving $l$, and produced by the limitation of lunar arguments to their linear development.

Finally, the difference with arguments corrected with the shift in phase does not exceed $1^{\prime \prime}$ over the 7000 years time span and is therefore non significant with respect to the background noise appearing in the comparisons with numerical integrations.

\section{Conclusion}

In this paper, we have presented a new solution for planetary perturbations of the Moon. This solution has been built taking advantage of the improvements of the planetary theory VSOP2000, and with a greater accuracy with respect to the ELP2000-82B solution for Moon's planetary perturbations. The comparisons revealed differences with long periodic terms and with their associated short periodic terms as expected. The adjustment of constants and orbital parameters to numerical integrations of the Jet Propulsion Laboratory DE403 and DE406 are under construction. The improvement of the analytical solution for the planetary perturbations should provide a lunar ephemeris obtained with a greater precision. The numerical complements remain still necessary for the comparisons to Lunar Laser Ranging observations.

Acknowledgements. The author would like to thank X. Moisson, M. Chapront-Touzé and especially J. Chapront for their many useful pieces of information, and their great help in numerical computations.

\section{References}

Chapront, J., Bretagnon, P., \& Mehl, M. 1975, Celest. Mech., 11,379

Chapront, J., \& Abu el Ata, N. 1977, A\&A, 55, 83

Chapront-Touzé, M., \& Chapront, J. 1980, A\&A, 91, 233

Chapront-Touzé, M. 1980, A\&A, 83, 86

Moisson, X. 1999, A\&A, 341, 318

Moisson, X. 2000, private communication

Moshier, S. L. 1992, A\&A, 262, 613

Simon, J. L., Bretagnon, P., Chapront, J., et al. 1994, A\&A, 282,663 